\title{
Breast Cancer in Lopburi, a Province in Central Thailand: Analysis of 2001-2010 Incidence and Future Trends
}

\author{
Somphob Sangkittipaiboon ${ }^{1}$, Atit Leklob ${ }^{1}$, Hutcha Sriplung ${ }^{2 *}$, Surichai Bilheem ${ }^{2}$
}

\begin{abstract}
Background: Thailand has come to an epidemiologic transition with decreasing infectious diseases and increasing burden of chronic conditions, including cancer. Breast cancer has the highest incidence rates among females throughout Thailand. This study aimed to identify the current burden and the future trends of breast cancer of Lopburi, a province in the Central Thailand. Materials and Methods: We used cancer incidence data from the Lopburi Cancer Registry to characterize and analyze the incidence of breast cancer in Central Thailand. With joinpoint and age-period-cohort analyses, the incidence of breast cancer in the province from 2001 to 2010 and project future trends from 2011 to 2030 was investigated. Results: Age-adjusted incidence rates of breast cancer in Lopburi increased from 23.4 to 34.3 cases per 100,000 female population during the period, equivalent to an annual percentage change of $4.3 \%$ per year. Both period and cohort effects played a role in shaping the increase in incidence. Joinpoint projection suggested that incidence rates would continue to increase in the future with incidence for women ages 50 years and above increasing at a higher rate than for women below the age of 50. Conclusions: The current situation where early detection measures are being promoted could increase detection rates of the disease. Preparation of sufficient budget for treatment facilities and human resources, both in surgical and medical oncology, is essential for future medical care.
\end{abstract}

Keywords: Breast cancer - cancer incidence - joinpoint - age period cohort model - Lopburi - Thailand

Asian Pac J Cancer Prev, 16 (18), 8359-8364

\section{Introduction}

In Thailand, an epidemiologic transition has occurred in terms of decreasing rates of mortality due to infectious diseases and increasing rates of chronic and metabolic conditions, including cancer. New cancer cases and death in less developed countries are estimated to be 57\% and $65 \%$ of the world cancer burden and new cases are predicted to rise $63 \%$ in less developed countries (Globocan 2012 - Home, n.d.). There is a strong correlation between Western lifestyle factors, such as diet and parity, and the incidence of breast cancer (Layde et al, 1989; Althuis, 2005; Cui et al, 2007; Kruk, 2007). Breast cancer poses a particular problem over the next decades as less developed countries are increasingly adopting characteristics of a Western lifestyle. Mammographic screening is limited to women who can financially access to it and are aware of breast cancer such as those who have family history of breast cancer. According to the Ministry of Public Health, breast self examination is the only method affordable and recommended to all public health care in the country to promote awareness and down-staging of the disease. Thus, to target resources to breast cancer prevention and control, incidence predictions are crucial.

Breast cancer incidence rates are increasing in all regions of Thailand (Sriplung et al, 2006; Khuhaprema et al, 2013). The estimated age-standardized incidence rate (ASR) of breast cancer in Thailand increased from 13.5 to 30.7 cases per 100,000 female population in 1990 and 2008 (Vatanasapt et al, 1995; Khuhaprema et al., 2013). The regions of Thailand vary dramatically in terms of population characteristics, risk factor exposures i.e. ethnic composition and diet, and incidence rates (Khuhaprema et al., 2012). Central Thailand consists of a population of unique ethnic and cultural make-up.

Lopburi is a province in the Central region border to the Northeastern region of Thailand (Lopburi Province - Wikipedia, n.d.). It occupies an area of 6,199.8 sq.km (Figure 1) and the population at 2010 census was 0.75 million. The province is subdivided into 11 districts. The main income of the province is from tourism and agricultural products.

Lopburi Cancer Hospital (formerly known as Lopburi Cancer Center) was established in 1995 to cover cancer control activities and treatment of cancer patients in the upper Central Thailand. Lopburi cancer registry was set up in 2000 to provide cancer statistics of the upper Central Thailand region (Health Care Service Region 4) especially in Lopburi province where the cancer center is located.

The aim of this study was to analyze the breast cancer 
incidence trends in the province by calendar year, birthcohort and age of diagnosis and to project female breast cancer rates in Lopburi province, the representative of Central Thailand through till 2030. The investigation in this study included incidence rates of female breast cancer data from the Lopburi registry from 2001 to 2010 using joinpoint regression and age-period-cohort models.

\section{Materials and Methods}

\section{Cancer registry and cancer case recruitment}

The Lopburi registry covers eleven districts in Central Thailand. The population of southern Thailand at the 2010 census was 0.75 million people of which 0.39 million were females (National Statistical Office, 2011). The registry actively ascertains cancer cases from various sources including community hospitals, private hospitals, and the Bureau of Policy and Strategies, Ministry of Public Health.

The number of undetected cases is difficult to estimate due to the preference of some patients to visit famous hospitals in Bangkok, the capital of Thailand. However, it is expected to be low since the registry has a close collaboration with the cancer registry of Bangkok run by the National Cancer Institute and used the Bangkok registry as one of its source of case identification. Female breast cancer cases were extracted from the registry database from 2001 through 2010 using ICD-10 codes C50.X. Information included age and date of diagnosis.

\section{Population denominators}

Population denominators to calculate incidence rates were estimated from the three population censuses surveyed by the National Statistical Office in 1990, 2000, and 2010 (National Statistical Office, 2012, 2002). The population denominators by both sexes for all districts were readily present in the censuses. Intercensus populations were estimated using a log-linear function between two consecutive censuses. The populations beyond 2010 were estimated and reported by the Office of the National Economic and Social Development Board (2013).

\section{Statistical analysis}

ASRs standardized to the world population proposed by Segi (1960), and later modified by Doll (1976), were computed for each particular year and then plotted to visually illustrate the trends. Person-years used as the denominators in the computation were calculated from census data (National Statistical Office, 2011). Agespecific incidence rates were calculated for eighteen age groups ranging from $0-4$, through $80-84$, and 85 years and older and ten calendar periods from 2001 to 2010 (at 1-year intervals).

We evaluated trends in incidence using the JoinpointRegression Program version 4.0.4 (National Cancer Institute, 2013). Joinpoint regression identifies statistically significant trend change points (joinpoints) and the rate of change (annual percent change) in each trend segment using a Monte Carlo permutation method (Kim et al., 2000). Analyses were conducted for all females, and then for females younger than 50, and females 50 years of age or older to determine the differences in incidence trends above and below the mean age of menopause (Thomas et al., 2001; Henderson et al., 2008).

Age-period-cohort (APC) regression models were used to investigate the effect of age, calendar year and birth-cohort on the incidence of breast cancer. Since the time period is only 10 years, age-specific incidence rates were calculated for 1-year rather than 5-year age groups. Though the use of 1-year interval had the limitation of high fluctuating rates, and caused wide confidence intervals, it allowed us to use the dataset with a short time period to visualize the trend, as well as being able to project the future incidence rates. We used the classical method which fits a log-linear model with a Poisson distribution to the observed data to estimate age, period and cohort effects in a multiplicative APC model as follows:

$$
\log [R(a, p)]=f(a)+g(p)+h(c) .
$$

Where the expected $\log$-incidence rates $R(a, p)$ is assumed to be equal to a linear combination of effects that adjusts for age $a$, period $p$ and birth-cohort $c$, where $c=p-a$. To address the non-identifiability problem of the APC models, two-effects models (age-period and agecohort) were first chosen and the remaining effect (cohort or period) was then identified to the respective model's residuals using natural splines to reduce random variation (Carstensen, 2012). These are referred to as the AP-C and AC-P models. The analysis of APC models was performed with the Epi package (Carstensen et al., 2013) for R statistical software version 3.1.2 (R Core Team, 2014).

We used the two independent methods to project the incidence rates of breast cancer in the Lopburi province; joinpoint and age-period-cohort model projections. Joinpoint: Each best-fit joinpoint model was separated into its linear and residual components. The residuals described the curvature while the linear component illustrated the secular drift of the trend. APC: The linear drift $(D)$ of period and cohort parameter was identified. With both methods, the incidence rates were extrapolated out to 2030. We also follow the cut trend system mentioned

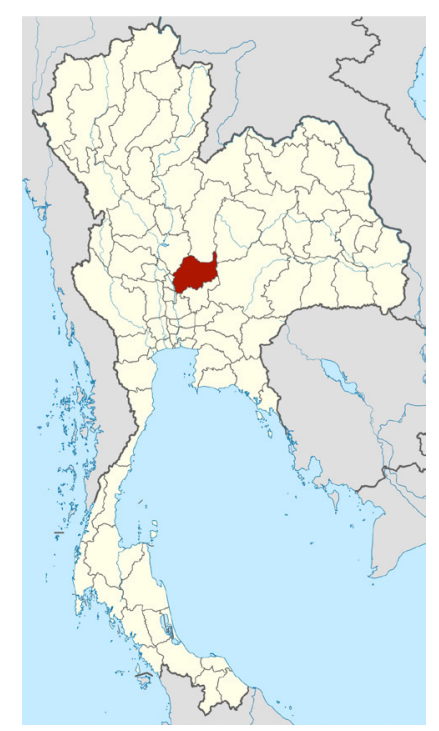

Figure 1. Map of Thailand. The province of Lopburi has been highlighted 
by Mistry et al. (2011) in projection by attenuation of the linear drift by $0 \mathrm{D}, 0.216 \mathrm{D}, 0.483 \mathrm{D}$, and $0.659 \mathrm{D}$ respectively, for the four future 5 -year projection periods.

\section{Results}

A total of 1,439 female breast cancer cases were diagnosed in Lopburi province from 2001 to 2010. Of these, 693 occurred in females under the age of 50 and 746 cases in females over the age of 50. Stage distribution was shown by each age group in Figure 2. The underlying trends in stage classification for both groups of patients showed a dramatic increasing percentage of cases in the
DOI:http://dx.doi.org/10.7314/APJCP.2015.16.18.8359 Trends of Breast Cancer Incidence in Lopburi, Central Thailand last 3-year period for localized cancers and a decrease in unknown cancers. The percentage of cases with unknown stage was rather stable through out the period from 2001 to 2010 among women below 50 years of age and 50 years and older, except the slightly lower percentage in the first 3 -year period among women below 50. The percentage of distant stage decreases while the localized stage appeared to be stable over the time period among women aged 50 and older, while it was not so true among the younger age groups.

The age group at peak incidence was around 50-54 and was about the same over the time periods (Figure 3 ). The second rise in age-specific incidence rates was observed
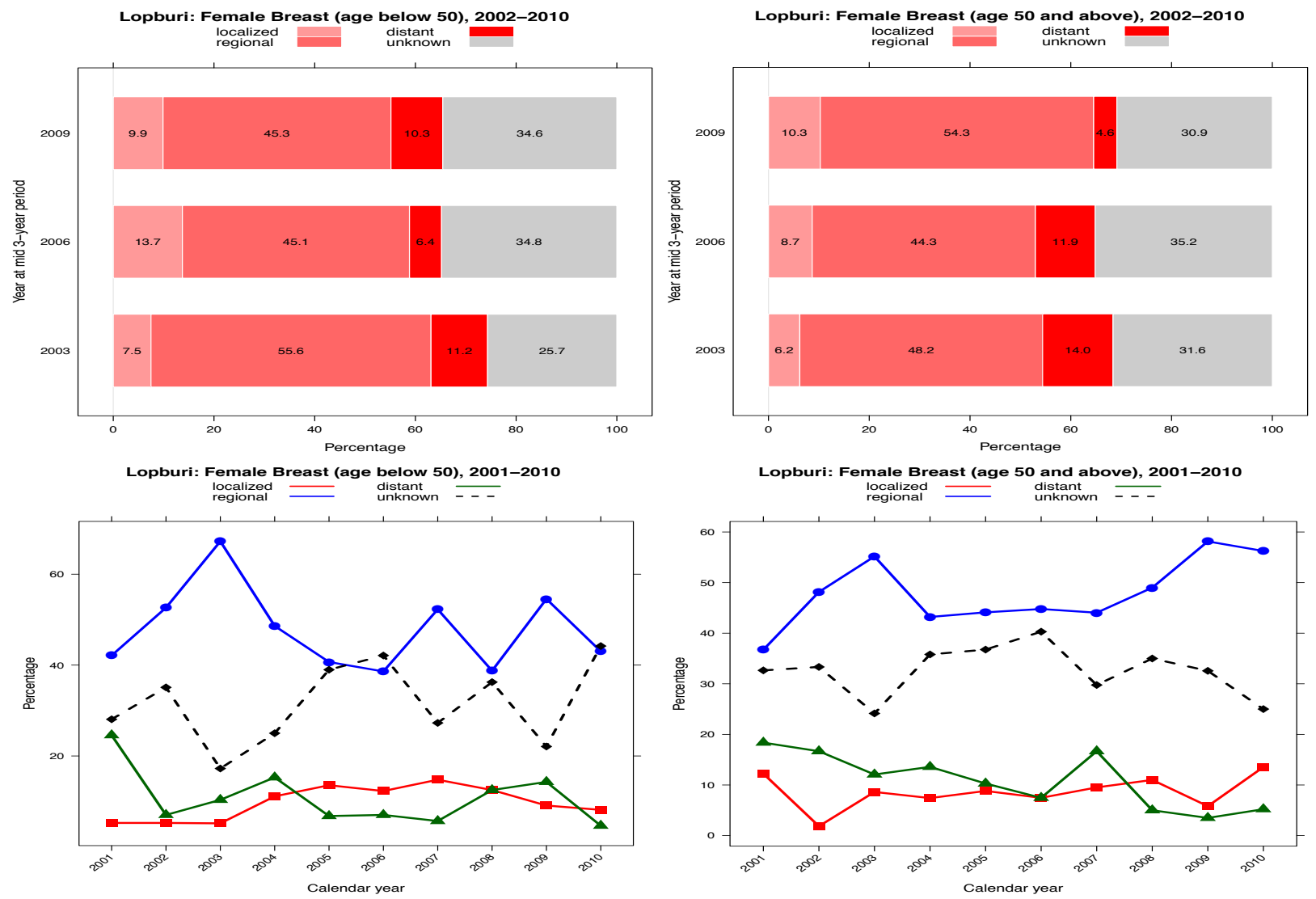

Figure 2. Stage Distribution Across 3-year Periods for a) Women Below the Age of 50 and b) Women at, or Above the Age of 50. Percentage of stage for each year for c) women below the age of 50 and d) women at or above the age of 50

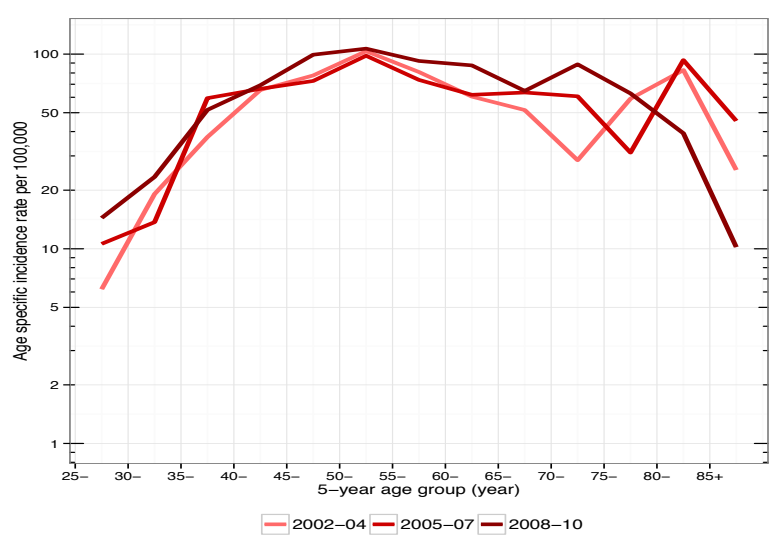

Figure 3. Age-specific Incidence Curves of Breast Cancer in Lopburi in three 3-year Periods from 200204, 2005-07, and 2008-10

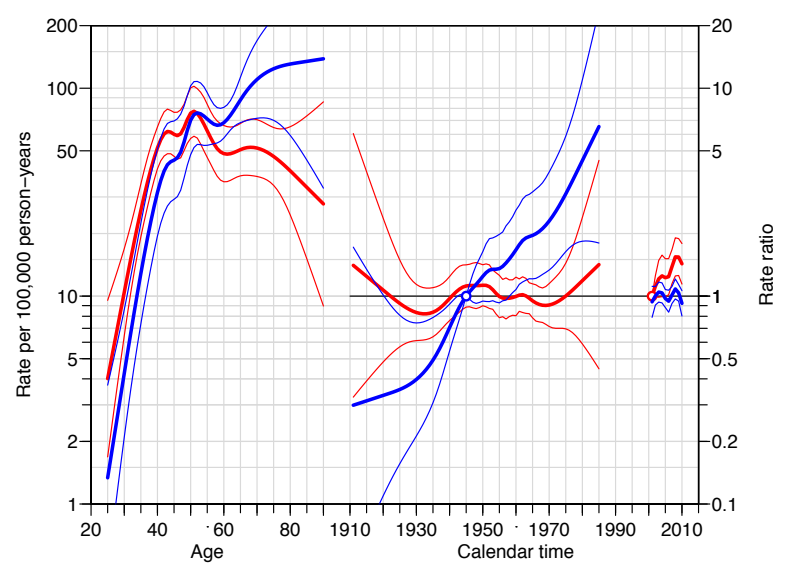

Figure 4. APC Trend Analysis. AC-P (blue) and AP-C (red) models for all females. Incidence rates are plotted in the $\log$ scale (left y-axis) 

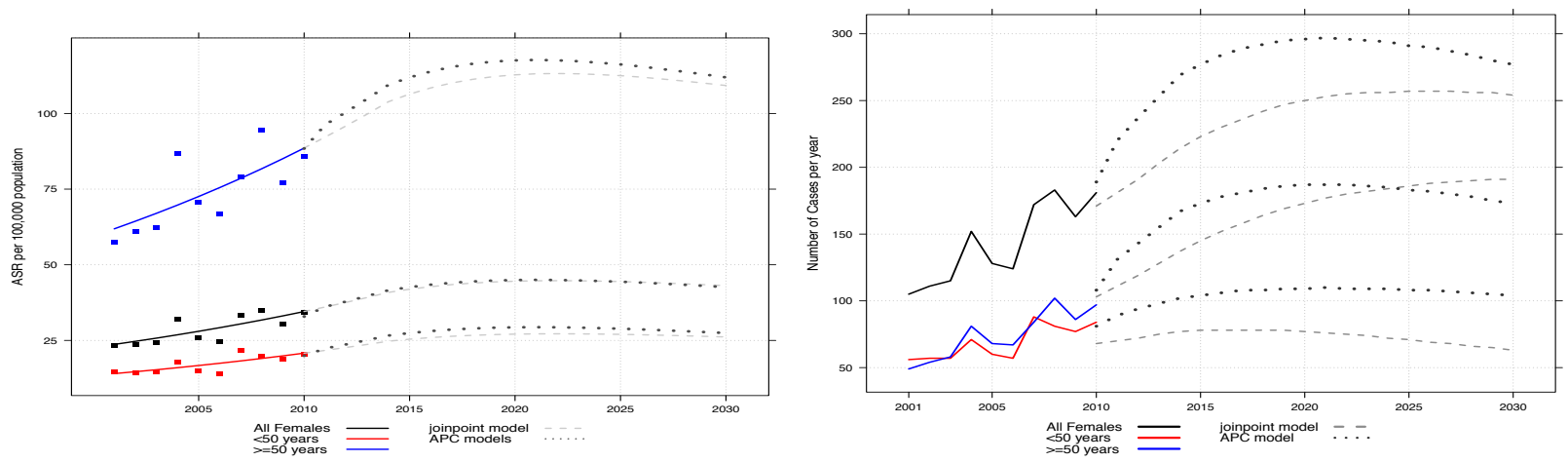

Figure 5. Breast Cancer Incidence Trend Projections to 2030. a) Rate projections using joinpoint (dashed lines) and ageperiod-cohort (dotted lines) models both for females 50 years and older are much higher than those of women age less than 50 years. b) Number of cases projected for all females are greater influenced by women aged 50 years and older than those less than 50 years around 70 to 84 in different periods.

Age-period-cohort analyses and joinpoint analysis

The AP-C and AC-P models of age-period-cohort trend analysis (Figure 4) showed rough linear trends in cohort and period across the calendar years. Thus, the overall increase in incidence was explained by the age-drift model with the drift parameter (coefficient) of 0.040 with $95 \%$ CI of 0.021-0.058.

Breast cancer incidence rates increased from an ASR of 23.4 in 2001 to an ASR of 34.3 cases per 100,000 personyears in 2010 (Figure 5). Zero join point model was the best fit for all ages and the two subgroups, women below 50 years of age and age 50 years and above. Overall, the incidence rate of female breast cancer increased at an annual percentage change (APC) of $4.3 \%$ (95\% CI: $1.4 \%$ $7.2 \%$ ) and $4.0 \%$ (95\% CI: $2.2 \%-5.9 \%$ ) by joinpoint and APC models, respectively.

The projection illustrated that the incidence rates would be continuously increasing in the future through 2020, and then slightly declining. However, we prefer a stable ASR from 2020 to 2030, thus, the highest ASR would be around 44.7 to 45.0 per 100,000 person-years for all ages, and 113.2 to 117.7 per 100,000 person-years among those aged 50 and above by joinpoint and APC methods, respectively (Figure 3).

As the proportion of elderly in the province also increases in the future, the number of patient would reach 297 cases per year by the APC models in 2020 and then dropped down to 277 cases per year in 2030. However, we might expect around 300 cases per year from 2020 by APC model while those aged 50 years and above would be 187 cases per year. In contrast, joinpoint model, which is less accurate in estimating the number of cases, predicts the number of around 277 cases per year in 2030 and the number of cases at the age of 50 years and above is predicted around 191. In summary, the cases aged 50 years and above would be around $70 \%$ by joinpoint method and around $63 \%$ by APC model.

\section{Discussion}

Breast cancer incidence in Lopburi has increased significantly since 2001 . This is likely due to a combination of changes in demographics and the risk profile of the population, as well as increases in surveillance and breast cancer awareness.

This shift in risk profiles of Thai women makes it necessary to focus on early detection and more awareness of breast cancer. As discussed in Virani et al. (2014), the breast cancer risk profile and screening of women in Thailand, the percentage of Thai women who were overweight, having diabetes, or hypertension found to be increased by the two consecutive health examination surveys in 2004 and 2009. Considering parity, the total fertility rate in Thai population has been gradually decreasing from 6 in 1970 to 3 in 1985, 2 in 1998 and 1.6 in 2010.

Projections from joinpoint regression model shows that the burden of breast cancer in the province will continue to rise in the near future, potentially reaching an incidence rate of about 62 cases per 100,000 population for all females in 2030. The ASR estimated from APC model was almost the same as that estimated from joinpoint model through 2030, in contrast, the number of patients estimated from APC model rose rapidly than that estimated from joinpoint model, but by 2020 , the estimates of the number of patients with both methods are not much different (Figure 5). However, because the estimates of number of cases by APC model used the 5-year age groups, the number is more accurate than the method used by joinpoint model where the sum of population in the two strata; 25-49 and 50-85+ age groups were used.

The increase could be partly affected by healthcare management and other cancer burden control measures which increase the early cancer detection rate by screening measures such as the use of breast self examination technique and mammography. Increase awareness of breast cancer in women in recent days could be playing role and the decrease in stage of the disease among patients aged 50 years and above would reflect the effect of both awareness and screening measures.

The down staging effect among women under 50 years was not clearly evident in Lopburi (Figure 2). This finding is contrast with that has been occurring in Songkhla in Southern Thailand (Virani et al, 2014). The reasons behind this phenomenon are to be investigated.

After an exponential increase in young ages, agespecific-incidence rates of breast cancer reached the peak around the age group of 50-54 (Figure 3). A slight drop in the incidence followed by a second peak can be seen 
in some Asian countries where the first peak has usually been reported around the age of 40-60 and the second at 85 and over, while bimodal curve is not common in western countries, the incidence usually peaks at the age of 70 and over (CI5I-X, n.d.).

Such the bimodal age effect is known as 'Clemmensen's hook' where incidence rates increase exponentially until around age of 50, and dip slightly before rising again at a lower rate (Figure 4). This phenomenon has been observed for breast cancer incidence and mortality in many countries especially in Asia, and is thought to be due to the overlap of rates between pre- and post-menopausal women (Waard et al., 1964; Anderson, 1974; Bouchardy et al., 2006).

The annual percent change obtained by the two models, jointpoint and APC, is not different. The increase in ASR of breast cancer in Lopburi by 4.0-4.3\% per year is similar to that observed in Southern Thailand (Virani et al., 2014). The deceleration of the increasing rates and numbers of cases seen in Figure 3 is the effect of cut trend procedure described above. The reason behind the cut trend procedure was described by Olsen et al. (2008).

Compared to a high income country, such as the United States where the ASR of breast cancer in CIV volume X was over 100 cases per 100,000 population (CI5I-X, n.d.). The ASR of breast cancer in Lopburi is also relatively low in comparison to other low- and middle-income countries (LMICs). The ASRs from 1998 to 2002 in Korea, Taiwan, Hong Kong and Singapore were reported by Shin et al. (2010) as 37.2, 59.7, 69.1 and 90.1 cases per 100,000 of a female population, respectively. Thailand is in transition to an increased lifespan and the shift in lifestyle characteristics of its people, thus, the increasing burden of breast cancer is observed. The rates seem to correspond to the degree of westernization of Asian countries.

The change in the proportion of breast cancer patients at the age of 50 years and over from $53 \%$ in 2010 to $78 \%$ in 2030 is a striking phenomenon which implies that postmenopausal patients are going to take a higher proportion among breast cancer patients. The phenomenon is also true in Songkhla where the shift over time towards regional and localized stages was striking and the proportion of distant metastatic cancers was gradually decreasing (Virani et al., 2014). It implies that the proportion of operable cases and patients who need hormonal and chemotherapy treatment is increasing as well. Therefore, the need for surgical and medical oncologists for breast cancer patients, and in fact, for other adenocarcinoma cancers will not only be a big constrain for health and medical care in Central Thailand, but for the country as a whole.

One of the limitations in this study is the fact that prevalence cases are usually registered as incidence cases in a newly established registry. It usually happens when a registry searching for new cancer cases from the hospital information system (HIS) fails to trace back to find the correct date of diagnosis which may be years before the date appears in the computerized HIS.

The absence of the shift in peak age of incidence towards younger age groups in Figure 4, which is inconsistent with the other registries with longer operation

time in Thailand (Vatanasapt et al, 1995; Deerassamee et al, 1999; Sriplung et al, 2003; Khuhaprema et al, 2007, 2010, 2012, 2013). The younger age at peak incidence among Asian population was also observed in other countries in Asia (Leong et al, 2010) and the shift in the age at peak incidence towards older age groups was also found in China (Fan et al, 2009).

Lopburi is located in Central Thailand and is not too far from Bangkok, the capital of Thailand, thus, cancer patients can by pass the referral system of the Ministry of Public Health to famous hospitals, especially that of medical schools in Bangkok. It is possible that there is some degree of under-registration in Lopburi cancer registry.

In conclusion, breast cancer in Central Thailand has been and will be continuously increasing. The majority of the patients in the future are among women aged 50 years and older. Primary prevention measures depend on the effectiveness of the control measures of metabolic disorder conditions, which is the general trend towards western lifestyle.

Screening measures and increased detection rate of early disease alone cannot reduce the increasing trend of the disease. And for these reasons the budget for treatment facilities, such as tertiary hospitals, with surgical facilities, and personnel in both surgical and medical oncology is going to be essential in the future.

\section{Acknowledgements}

We would like to thank Lopburi cancer registry for information on breast cancer data. Dr. Sriplung acknowledges the support from the National Research University Fund, Prince of Songkla University (MED580635S).

\section{References}

Althuis MD (2005). Global trends in breast cancer incidence and mortality 1973-1997. Int J Epidemiol, 34, 405-12.

Anderson DE (1974). Genetic study of breast cancer: identification of a high risk group. Cancer, 34, 1090-7.

Bouchardy C, Morabia A, Verkooijen HM, et al (2006). Remarkable change in age-specific breast cancer incidence in the Swiss canton of Geneva and its possible relation with the use of hormone replacement therapy. BMC Cancer, $\mathbf{6}, 78$.

Carstensen B (2012). Statistical Analysis in the Lexis Diagram: Age-Period-Cohort Models. Gentofte, Denmark: Steno Diabetes Center.

Carstensen B, Plummer M, Laara E, Hills M (2013). Epi: a package for statistical analysis in epidemiology, $\mathrm{R}$ package version 1.1.49, CRAN.

CI5I-X (n.d.).URL http://ci5.iarc.fr/CI5I-X/old/table4.asp?regi stry $=8402499 \&$ female $=2 \&$ volume $=1020032007 \&$ submit $=$ Execute (accessed 5.8.15).

Cui X, Dai Q, Tseng M, Shu X-O, Gao Y-T, Zheng W (2007). Dietary patterns and breast cancer risk in the shanghai breast cancer study. Cancer Epidemiol Biomark Prev, 16, 1443-8.

Deerassamee S, Martin N, Sontipong S, et al (eds) (1999). Cancer in Thailand vol. II, 1992-1994. IARC Technical Report No. 34, Lyon, France: International Agency for Research on Cancer.

De Waard F, Baanders-Vanhalewijn EA, Huizinga J (1964). 
Somphob Sangkittipaiboon et al

The bimodal age distribution of patients with mammary carcinoma; evidence for the existence of 2 types of human breast cancer. Cancer, 17, 141-51.

Doll R (1976). Comparison between Registries. AgeStandardized Rates, in: Waterhouse JAH, Muir CS, Correa P, Powell J (eds.), Cancer Incidence in Five Continents, Vol. III. IARC Scientific Publications No. 15. Lyon, France: International Agency for Research on Cancer, pp. 453-459.

Fan L, Zheng Y, Yu K-D, et al (2009). Breast cancer in a transitional society over 18 years: trends and present status in Shanghai, China. Breast Cancer Res Treat, 117, 409-416.

Globocan 2012 - Home (n.d.). URL http://globocan.iarc.fr/ Default.aspx (accessed 4.8.15).

Henderson KD, Bernstein L, Henderson B, Kolonel L, Pike MC (2008). Predictors of the timing of natural menopause in the Multiethnic Cohort Study. Am J Epidemiol, 167, 1287-94.

National Cancer Institute (2013). Joinpoint Regression Program, Version 4.0.4, Statistical methodology and applications branch. Surveillance Research Program, National Cancer Institute. from https://surveillance.cancer.gov/joinpoint/ download (accessed 5.6.13)

Khuhaprema T, Attasara P, Sriplung H, Wiangnon S, Sangrajrang S (eds.) (2013). Cancer in Thailand. Vol VII, 2007-2009. Bangkok, Thailand: National Cancer Institute.

Khuhaprema T, Attasara P, Sriplung H, Wiangnon S, Sumitsawan Y, Sangrajrang S (eds.) (2012). Cancer in Thailand. Vol VI, 2004-2006. Bangkok, Thailand: National Cancer Institute.

Khuhaprema T, Srivatanakul P, Attasara P, Sriplung H, Wiangnon S, Sumitsawan Y (eds.) (2010). Cancer in Thailand. Vol V, 2001-2003. Bangkok, Thailand: National Cancer Institute.

Khuhaprema T, Srivatanakul P, Sriplung H, Wiangnon S, Sumitsawan Y, Attasara P (eds.) (2007). Cancer in Thailand. Vol IV, 1998-2000. Bangkok, Thailand: Bangkok Medical Publisher.

Kim HJ, Fay MP, Feuer EJ, Midthune DN (2000). Permutation tests for joinpoint regression with applications to cancer rates. Stat Med, 19, 335-51.

Kruk J (2007). Association of lifestyle and other risk factors with breast cancer according to menopausal status: a case-control study in the Region of Western Pomerania (Poland). Asian Pac J Cancer Prev, 8, 513-24.

Layde PM, Webster LA, Baughman AL, et al (1989). The independent associations of parity, age at first full term pregnancy, and duration of breastfeeding with the risk of breast cancer. J Clin Epidemiol, 42, 963-73.

Leong SPL, Shen Z-Z, Liu T-J, et al (2010). Is breast cancer the same disease in Asian and Western countries? World $J$ Surg, 34, 2308-24.

Lopburi Province - Wikipedia, the free encyclopedia (n.d.). URL http://en.wikipedia.org/wiki/Lopburi_Province (accessed 4.9.15).

Mistry M, Parkin DM, Ahmad AS, Sasieni P. (2011) Cancer incidence in the United Kingdom: projections to the year 2030. Br J Cancer, 105, 1795-803.

National Statistical Office (2012). 2010 Population and Housing Census. Bangkok, Thailand: National Statistical Office.

National Statistical Office (2011). Population Census, 2010, Lopburi. Bangkok, Thailand: National Statistical Office.

National Statistical Office (2002). 2000 Population and Housing Census. Bangkok, Thailand: National Statistical Office.

National Statistical Office (1992). Advance Report: 1990 Population and Housing Census. Bangkok, Thailand: National Statistical Office.

Office of the National Economic and Social Development Board (2013). Population Projections for Thailand 2010-2040. Bangkok, Thailand: Office of the National Economic and Social Development Board.
R Core Team (2014). R: A language and environment for statistical computing. Vianna, Austria: R Core Team.

Segi M (1960). Cancer mortality for selected sites in 24 countries (1950-57). Sendai, Japan: Department of Public Health, Tohoku University of Medicine.

Shin H-R, Joubert C, Boniol M, et al (2010). Recent trends and patterns in breast cancer incidence among Eastern and Southeastern Asian women. Cancer Causes Control, 21, 1777-85.

Sriplung H, Sontipong S, Martin N, et al (eds.) (2003). Cancer in Thailand. Vol III, 1995-1997. Bangkok, Thailand: Bangkok Medical Publisher.

Sriplung H, Wiangnon S, Sontipong S, Sumitsawan Y, Martin $\mathrm{N}$ (2006). Cancer incidence trends in Thailand, 1989-2000. Asian Pac J Cancer Prev, 7, 239-44.

Thomas F, Renaud F, Benefice E, de Meeüs T, Guegan JF (2001). International variability of ages at menarche and menopause: patterns and main determinants. Hum Biol, 73, 271-290.

Vatanasapt V, Martin N, Sriplung H, et al (1995). Cancer incidence in Thailand, 1988-1991. Cancer Epidemiol Biomark Prev, 4, 475-83.

Virani S, Sriplung H, Rozek LS, Meza R (2014). Escalating burden of breast cancer in southern Thailand: analysis of 1990-2010 incidence and prediction of future trends. Cancer Epidemiol, 38, 235-43. 